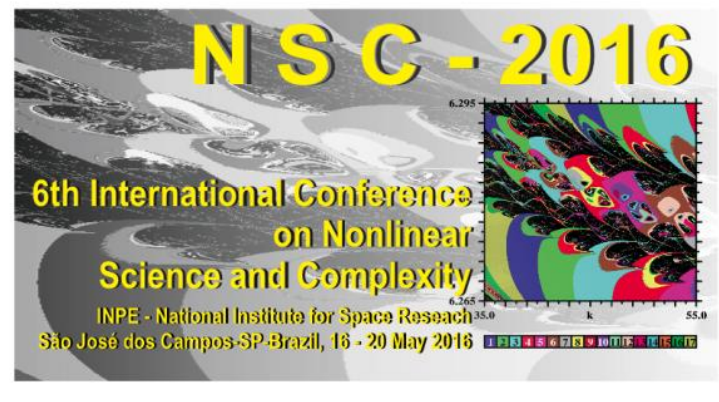

INPE - National Institute for Space Research

São José dos Campos - SP - Brazil - May 16-20, 2016

\title{
3DBMO: A TIME SERIES CANONICAL GENERATOR TO STUDY THE PSD DIMENSIONAL DEPENDENCE IN COMPLEX PHYSICAL SYSTEMS
}

\author{
Paulo Giovani de Faria Zeferino ${ }^{1}$, Reinaldo Roberto Rosa ${ }^{2}$, Murilo da Silva Dantas ${ }^{3}$ \\ 1 CAP/National Institute for Space Research - INPE, São José dos Campos, Brazil,pg_faria@ yahoo.com.br \\ 2 LAC/National Institute for Space Research - INPE, São José dos Campos, Brazil, rrrosa.inpe@gmail.com \\ 3 Federal Institute of Education, Science and Technology of São Paulo - IFSP, Jacareí, Brazil, murilo.dantas@ifsp.edu.br
}

\begin{abstract}
The study of density, magnetic and electric field fluctuations in real systems, such as nonlinear processes in the solar, magnetospheric and ionospheric environments, is generally analyzed using Power Spectrum Density (PSD) which is calculated from onedimensional data in the form of time series. In this work we present a new simulation device of multi-dimensional harmonic mechanical oscillations (we call 3DBMO) for generate robust time series from selected elements, where the spectral analysis is used to determine the hypothesis of dependence variation of the power spectrum according to the structure of the generated system. The importance of this study is discussed into the context of complex physical systems as nonlinear oscillators, fluids and plasmas, to which the correspondent dynamics is usually characterized by using PSD and related scaling exponents.
\end{abstract}

keywords: Complex Dynamics, Time Series Analysis, Coupled Oscillators System, Modeling, Numerical Simulation and Optimization.

\section{INTRODUCTION}

The study of plasma oscillations in 3D real systems (e.g.: nonlinear processes in the solar plasma, magnetospheric and ionospheric) are usually analyzed from one-dimensional data in the form of time series [1]. Usually, the classification of nonlinear physical processes (e.g.: chaos, reaction-diffusion and turbulence) to study and monitoring of Space Weather is based on spectral indices calculated from the power spectrum $P(k) \approx k^{a}$, which expresses a correlation law between the fluctuation ranges from the $A(t)$ time series [2,3]. A key issue in this approach relates to the dependence of the spectral index in relation to the dimensionality of the system. Given a system whose variable $A(t)$ (1D) is generated from a physical process that occurs in higher dimensions (2D or
$3 \mathrm{D})$, there is some dependence on the value in relation to the dimensionality of the process?

In this paper we propose a system composed by $3 \mathrm{D}$ oscillators, which will be used to verify the dependencies between canonical time series. This approach allows to study the respective time series and its power spectrum, varying the dimensionality and the number of elements of the system, as well their basic physical properties.

\section{PURPOSE}

The prototype for 3D simulations is based on the basic physics properties of a mass-spring classic system, which identifies the mass $m$ of the element, its position to an equilibrium point $x=0$ and the spring constant $k$ [4], as can be seen in Figure 1. Note that it is possible to obtain a time series for the mass $m$ for both their position in time, as to its instantaneous velocity.

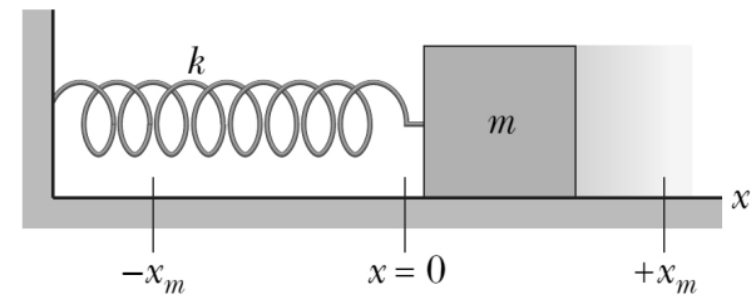

Figure 1 - Example of an harmonic oscillator system, with a mass $m$ connected to a spring of constant $k$.

The purpose of this work is to develop a prototype for the simulation of multi-dimensional harmonic oscillations. The main objective of the first prototype containing three particles in $1 \mathrm{D}$, nine particles in $2 \mathrm{D}$ and twenty seven particles in 3D is to generate and analyze time series of position and speed of selected elements from the simulator. The values that constitute these time series are generated synthetically and may be influenced 
by multi-dimensional disturbances through a system of coupled harmonic oscillators. In Figure 2 we can see an example of the 3D structure generated by the system, where the elements are starting the simulation. Our goal is to simulate several conditions to excite the system behavior, getting time series data for spectral analysis.

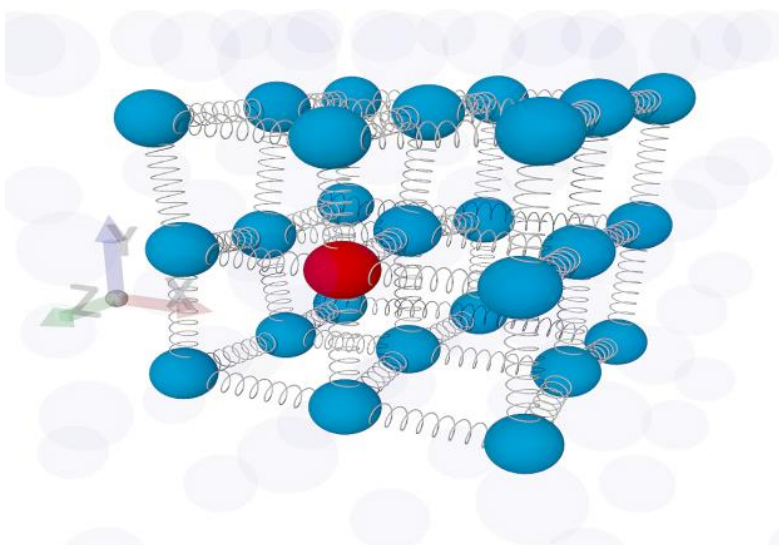

Figure 2 - 3D structure generated by the simulator for 27 elements. In this case, the system is starting the simulation and the red element will generate the desired data.

\section{METHODS}

This simulator was created using the Python language, with some of his features [5, 6], and the system is responsible for generating time series containing from $10^{4}$ points. The user can set various parameters such as the size of the structure, the quantity of elements comprising the oscillators system, the weight for these elements, the spring constant and the number of points that he wishes to perform the simulation. Each simulation will generate a data set that will be use to future analysis. This analysis will involve the power spectrum calculation, and the goal is to check the dependencies between the several dimensions when studying the same element among the dimensional structure (i.e. 1D, 2D and 3D). Figure 3 shows a basic flowchart of the operation of the system.

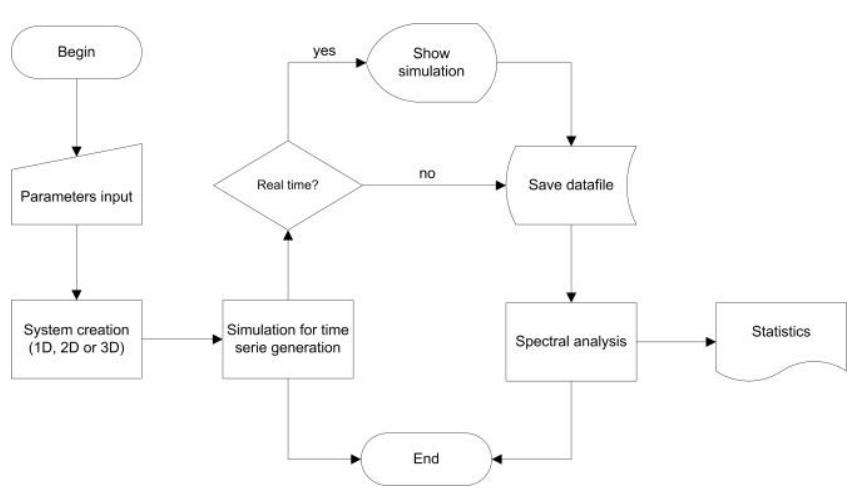

Figure 3 - Basic flowchart for system operation.

\section{RESULTS}

Each element present in the structure forming coupled oscillators system can generate data in a time series format. These data are stored in files which may be used for further analysis. Since the objective is to check the variation in the dependence of an element according to the size of the structure in which it lies, the analysis involves calculation of the power spectrum. Figure 4 shows spectral analysis of the values obtained for a time series containing $5 \cdot 10^{4}$ points. Parameters used for test are listed in Table 1.

\section{Table 1 - Test parameters for system generated with $5 \cdot 10^{4}$ points and spring constant $k=1.50$.}

\begin{tabular}{|l|c|}
\hline \multicolumn{1}{|c|}{ Parameter } & Value \\
\hline 3D space size & 3 \\
\hline Total of elements & 3 \\
\hline Distance between masses & 1.00 \\
\hline Mass radius & 0.30 \\
\hline Mass weight & 1.50 \\
\hline Mass to generate data & 23 \\
\hline Spring constant & 1.50 \\
\hline Total of points & 50000 \\
\hline
\end{tabular}
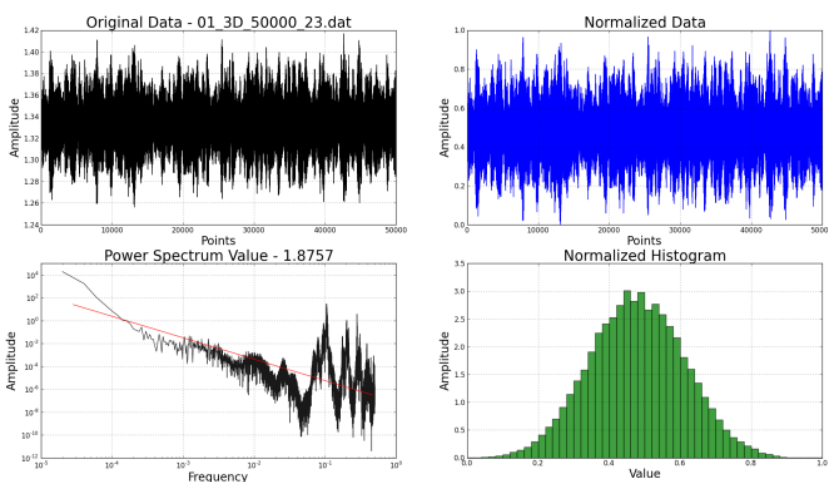

Figure 4 - Power spectrum for a single element in a 3D structure generated with the parameters listed in Table 1.

The 3D model created by simulator using the parameters listed in Table 1 can be visualized in Figure 5. Here, we can realize that the model structure has changed little during the simulation run.

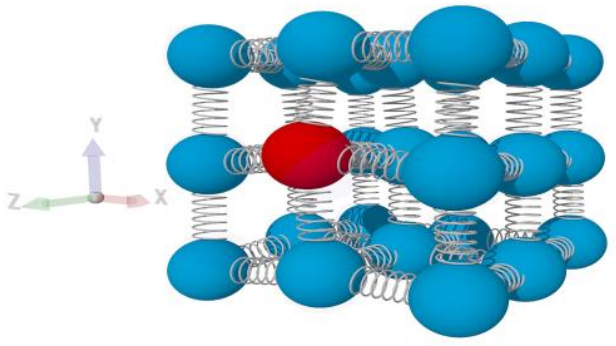

a

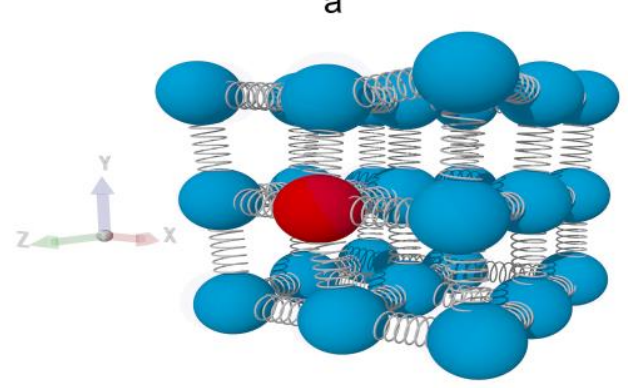

b

Figure 5 - 3D model in (a) start and (b) end of simulation, using the parameters listed in Table 1. 
However, when performing some changes in the input parameters, we can obtain interesting results. For example, changing the spring constant $k$ from 1.50 to 1.65 , as listed in Table 2, the model performs a chaotic behavior, which contributes to destroy the 3D structure at iteration 1415. Figure 6 displays the result of the power spectrum calculation for the moment when this situation occurs.

Table 2 - Test parameters for system generated with $5 \cdot 10^{4}$ points and spring constant $k=1.65$.

\begin{tabular}{|l|c|}
\hline \multicolumn{1}{|c|}{ Parameter } & Value \\
\hline 3D space size & 3 \\
\hline Total of elements & 3 \\
\hline Distance between masses & 1.00 \\
\hline Mass radius & 0.30 \\
\hline Mass weight & 1.50 \\
\hline Mass to generate data & 23 \\
\hline Spring constant & 1.65 \\
\hline Total of points & 50000 \\
\hline
\end{tabular}
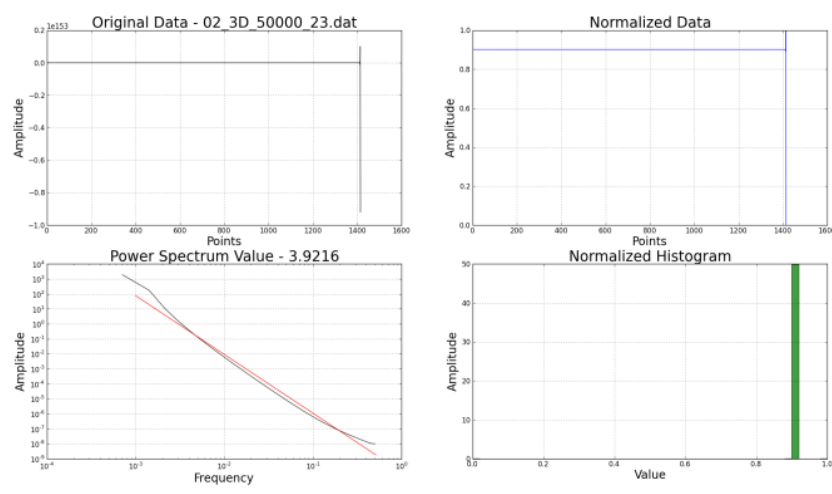

Figure 6 - Power spectrum for a single element in a 3D structure generated with the parameters listed in Table 2 .

Looking at the data generated during the simulation to $k=1.65$, it can be noted that the values began to diverge from iteration 1256 . Thus, the values generated during the iterations number 1257 to 1415 were removed, and the amount of the power spectrum was recalculated. The result can be seen in Figure 7.
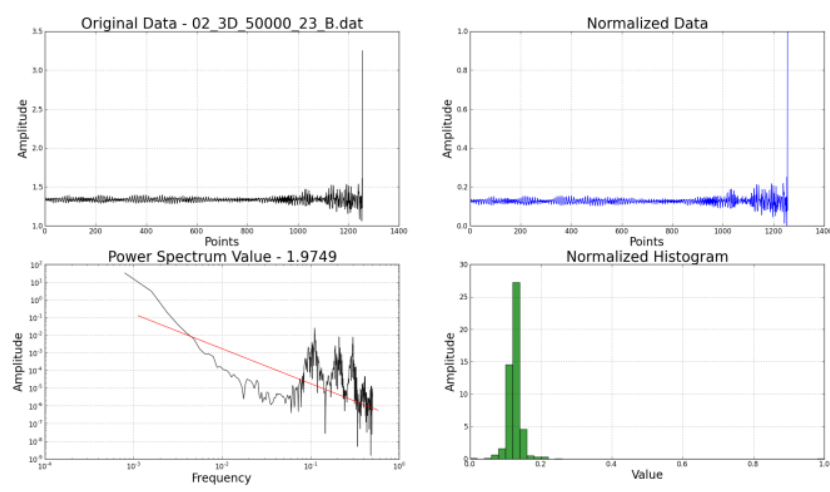

Figure 7 - Power spectrum for data generated with the parameters listed in Table 2, when removing divergent values.

Although we have a peak in the last position for amplitude values shown in Figure 7 , the data that were removed have very different values of average. Table 3 shows a comparison of the statistical moments calculated for each file on values of Figures 4, 6 and 7. Here, we can notice a great difference between the values obtained in Figure 6.

Table 3 - Statistical moments for the data of Figures 4, 6 and 7.

\begin{tabular}{|l|c|c|c|}
\hline & Figure 4 & Figure 5 & Figure 6 \\
\hline Min & 1.2561 & $-9.210610731 \mathrm{e}+152$ & 1.0586 \\
\hline Max & 1.4168 & $1.005776949 \mathrm{e}+152$ & 3.2506 \\
\hline Mean & 0.4787 & 0.9010 & 0.1259 \\
\hline Median & 0.4782 & 0.9016 & 0.1254 \\
\hline PSD & 1.8757 & 3.9216 & 1.9749 \\
\hline
\end{tabular}

It's possible to realize what happens to the 3D model structure for the data listed in Table 2 through the images in Figure 8. In this case, although in the beginning of the simulation the model had a cohesive structure, it is possible to observe the chaotic behavior started in the iteration number 1256. For the analysis, the goal is to determine whether some kind of change occurs in the spectral range of this time series generated by the $3 \mathrm{D}$ model.

The spectral analysis can be used to obtain the frequency of data [7]. The power spectrum provides another useful way to discern the fundamental harmonics of seemingly random signals [8]. For this, we use a Fast Fourier Transform (FFT) to obtain the power spectrum values for our time series data generated by the $3 \mathrm{D}$ model of coupled harmonic oscillators. However, this is not the only technique that can be used. For future work, the dependence of the power spectrum with the variation of the size of the system can be tested by applying other algorithms such as Detrended Fluctuation Analysis (DFA) and Global Wavelet Spectrum (GWS).

\section{CONCLUSION}

The importance of this study is discussed in the context of plasma density fluctuations analyzed by the power spectrum measured from a time series in various systems related to space weather.

When analyzing a data file, we usually do not have all information about how the data were collected. In real scenarios, an element can be influenced by others and this aspect can influence the analysis result. Depending on the situation, it is very hard to collect data. Thus, by using a controlled environment like this one proposed in this paper, scientists can be able to simulate several situations.

Through the analysis of the data collected in these scenarios it is expected that will be possible to find correlations indicating how the data was obtained. So, this study may help scientists during the execution of their activities.

\section{ACKNOWLEDGMENTS}

The author would like to thank the support of the Federal Institute of Education, Science and Technology of São Paulo (IFSP) - Campus Campos do Jordão and Campus Jacareí and National Institute for Space Research (INPE). 


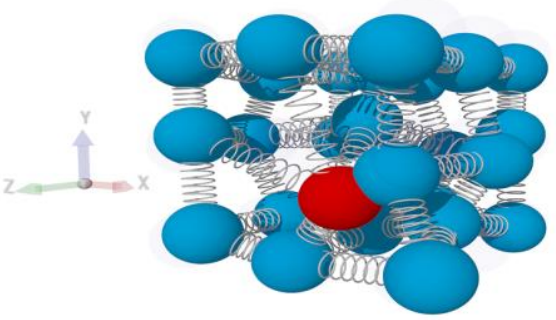

a

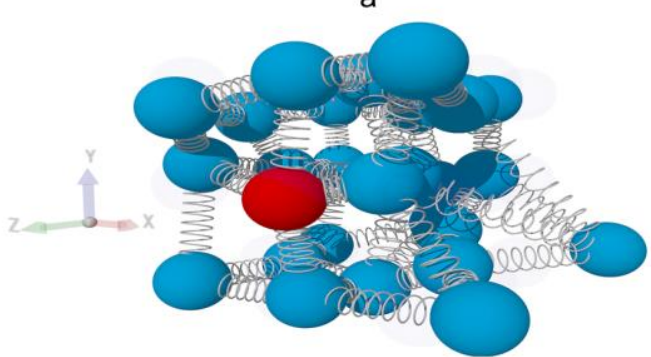

b

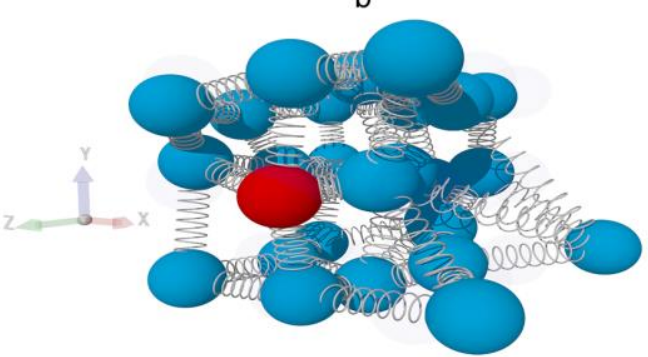

C

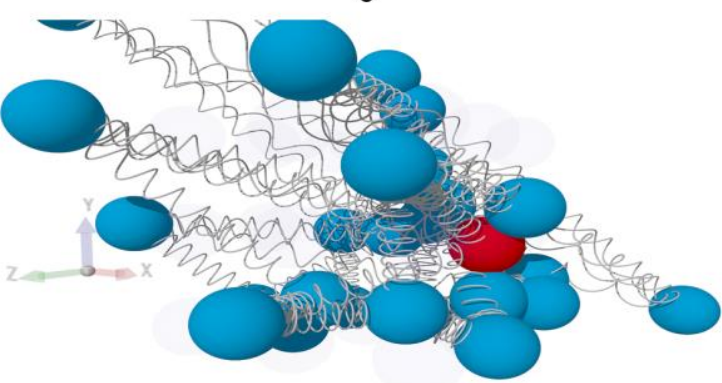

d

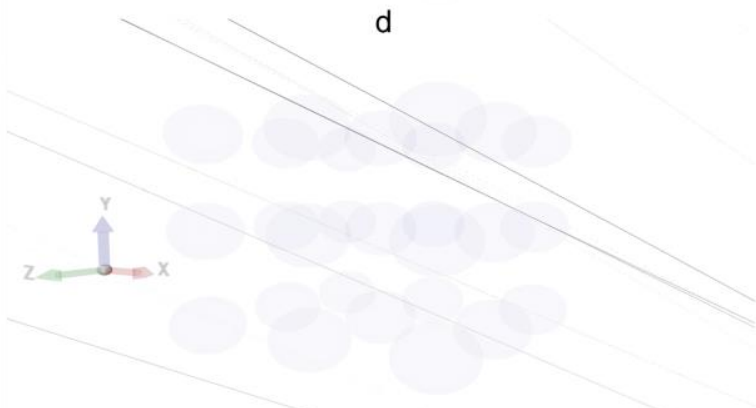

e

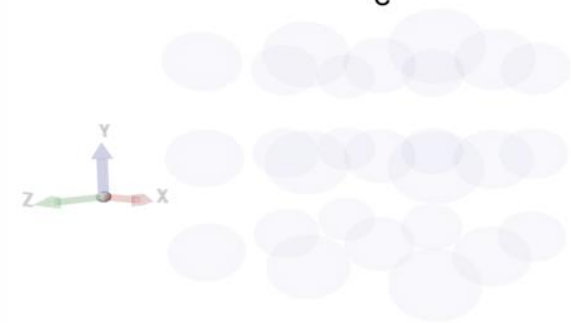

f
References

[1] R. O. Dendy, "Plasma Dynamics" on Oxford University Press Inc., 11-168, United States of America, 1990.

[2] A. Hanslmeier, "The Sun and Space Weather" on The Netherlands, Springer, 1-272, Dordrecht, The Netherlands, 2007.

[3] T. B. Veronese, R. R. Rosa, M. J. A. Bolzan et al., "Fluctuation Analysis of Solar Radio Bursts Associated with Geoeffective X-class Flares" on Journal of Atmospheric and Solar-Terrestrial Physics, v.73, 1311-1316, 2011.

[4] J. Walker, D. Halliday, R. Resnick, "Fundamentals of Physics: Forecasting and Control" on John Wiley \& Sons Inc., 386-403, United States of America, 2014.

[5] VPython, "VPython 3D Programming for Ordinary Mortals" [ONLINE] Available at: http://vpython.org/index.html. [Accessed 09 February 16].

[6] E. Jones, T. Oliphant, P. Petersons et al., "SciPy: Open Source Scientic Tools for Python" [ONLINE] Available at: http://www.scipy.org/.[Accessed 09 February 16].

[7] P. A. Morettin, C. M. C. Toloi, "Análise de Séries Temporais" on Editora Blucher, 435-476, São Paulo, Brasil, 2006.

[8] S. C. Chapra, "Métodos Numéricos Aplicados com Matlab para Engenheiros e Cientistas" on AMGH Editora Ltda., 399-402, Porto Alegre, Brasil, 2013. 